\title{
Call Forwarding-Based Active Probing for POTS Fault Isolation
}

\author{
Chi-Shih Chao $\cdot$ Maitreya Natu $\cdot$ Adarshpal S. Sethi
}

Published online: 19 September 2007

(C) Springer Science+Business Media, LLC 2007

\begin{abstract}
To ensure high availability of telephone services, there is considerable interest in network management activities to develop competent fault management mechanisms. In this paper we investigate, examine, and try to adapt the current active probing technologies to fault isolation and problem determination of telecommunication networks. The unfitness, issues, and difficulties while adopting these IP-networking probing methods into fault isolation of telecommunication networks are addressed and elaborated. For achieving our goal, call-forwarding which is a dedicated function (or feature) in telecommunication networks is utilized to overcome the unfitness and difficulties while applying probing technologies to fault isolation of telecommunication networks. Numerous experiments with different probe sets, probe stations, total probe lengths, network topologies, and probing algorithms are conducted and analyzed to validate our strategy. From the results of our experiments, we can find that phone call-forwarding can not only facilitate the fault isolation with the existing active probing techniques, but also
\end{abstract}

Prepared through collaborative participation in the Communications and Networks Consortium sponsored by the U.S. Army Research Laboratory under the Collaborative Technology Alliance Program, Cooperative Agreement DAAD19-01-2-0011. The U.S. Government is authorized to reproduce and distribute reprints for Government purposes notwithstanding any copyright notation thereon.

C.-S. Chao $(\bowtie)$

Department of Communications Engineering, Feng Chia University, No. 100 Wenhwa Rd., Seatwen, Taichung 40724, Taiwan, ROC

e-mail: cschao@fcu.edu.tw; chao@ieee.org

M. Natu A. S. Sethi

Department of Computer and Information Sciences, University of Delaware, Newark, DE 19716, USA

M. Natu

e-mail: natu@mail.cis.udel.edu

A. S. Sethi

e-mail: sethi@mail.cis.udel.edu 
increase the system flexibility on the installation of probe stations. Meanwhile, on the basis of our experimental results, several practical suggestions are also provided for system implementation.

Keywords Phone call-forwarding · Fault isolation · Active probing · Dependency matrix · Diagnostic power - Telecommunication networks

\section{Introduction}

As we depend more on networks today, network faults and downtime become very costly. Thus, the development of a practical and effective system for network fault diagnosis becomes a critical task. Numerous well-known products for fault management and fault isolation are available in the market [1]. For example, SMARTS's Distributed Event Correlation System (DECS), using Yemini's Codebook correlation [2], has the capability of handling noisy data-the lost, false, or corrupted symptomatic information. IBM's NetFACT operates via defining the alarm domain theory and constructing a normalized model of network configuration [3]. Other similar event-correlation-based fault management systems include: AT\&T Bell Laboratories' ECXpert, GTE Laboratories' IMPACT, OSI's NetExpert, and HP's Event Correlation Services (ECS). Additionally, there are also several renowned research results in the integration of network fault diagnosis with event correlation in the past few years. Rouvellou and Hart [4] adapt Probabilistic Finite State Machines (PFSMs) for specifying the behavior of faults in ANS (Advanced Network and Services, Inc.)/NSF T3 networks. Two inference algorithms based on the PFSM model are also proposed to deal with the corrupted and incomplete data. Schwartz [3] proposes the concept of alarm domains and uses it with several developed heuristic algorithms to complete effective fault isolation. Chao [5] introduces a feasible framework for temporal event correlating and filtering to identify faults on a LAN.

However, as networks continue to grow in size and complexity, system administrators are faced with an ever-increasing volume of event data, and tasks such as fault localization and problem determination become more difficult. As a result, tools are needed that can assist in performing these management tasks by responding quickly and accurately to the large number of events and alarms that are usually generated by even a single fault. Probing offers the opportunity to develop an approach to problem determination that is more active than traditional event correlation and other methods [6,7]. A probe is a program that executes on a particular machine (called a probe station) by sending a command or transaction to a server or network element and measuring the response. Probing technology is widely used to measure the quality of network performance, often motivated by the requirements of service-level-agreements (SLA). Examples of probing technology include the T. J. Watson EPP technology [8] and the Keynote product [9]. Regarding the isolation of network faults using probing technology, to date, the best known and most feasible methods are proposed by Brodie et al. [10, 11]. They present a framework for addressing the issue and implement algorithms that exploit 
interactions between probe paths to find a small collection of probes that can be used to locate faults. Small probe sets are desirable in order to minimize the costs imposed by probing, such as additional network load and data management requirement. The results show that although finding the optimal collection of probes is expensive for large networks, their efficient approximation algorithms can be used to find a nearly-optimal solution set.

In this paper we adapt the methods and algorithms proposed by Brodie et al. [10] to fault isolation of telecommunication networks as a case study in reality. To do so, first, the issues and difficulties while adopting probing technology into fault isolation of telecommunication networks are described. The goals for our system development are also indicated as the reference of our system design. In this process, we identify the presence of a special feature of telecommunication networks, namely call-forwarding, which has the potential of aiding the task of fault isolation via active probing. Such a feature is not usually available in general data networks and hence it has not been considered in the fault isolation approaches developed until now. We discuss how call-forwarding can be included in the process of fault isolation. Later, based on the concepts of active probing, we apply phone call-forwarding in the three algorithms proposed by Brodie et al. to achieve the goals of our system development for fault isolation. Next, results of an experimental study are presented to apply these techniques to fault isolation of Taichung City and Taichung County POTS's, and a multi-perspective analysis is done to check the fault isolation performance of our work. The rest of this paper is organized as follows: in Sect. 2, the issues and difficulties for the adaptation of probing technology to fault isolation of telecommunication networks are described while the goals of our system development are also mentioned. In Sect. 3, the concepts of active probing are described first. Three current widely-used active probing algorithms developed by Brodie et al. with the associated framework are also specified. On the basis of the concepts of active probing, we apply phone callforwarding in these three algorithms to achieve the goals of our system development for fault isolation in telecommunication networks. Section 4 shows and analyzes the results of experiments to realize the performance of our work and Sect. 5 concludes the paper with future works.

\section{Issues for Active Probing in POTS}

In this section, we will mainly describe the issues and difficulties that arise while adopting probing technology into fault isolation of telecommunication networks. In addition, the goals for our system development are also formulated as the reference of our system design.

\subsection{System Architecture and Probing Technology}

Before discussing the core of our problems, let us have a glance at the hierarchical architecture and operational characteristics of traditional telecommunication 
switching networks. Figure 1 shows a conceptual architectural hierarchy of a telecommunication network. As shown in Fig. 1, a typical telecommunication network consists of three tiers of switching systems. The lowest tier of the telecommunication hierarchy comprises EOs (End Offices, also called Local Switches) which are in charge of collecting the local loops of the designated geographical regions. MSs (tandeM Switches) in the middle tier are responsible for routing and relaying the tones among the switches. At the top tier of the hierarchy, TSs (Toll Switches or Long Distance Offices) are equipped to switch not only the phone calls within the hierarchy, but also inter-hierarchy phone calls (also called long-distance phone calls). Typically, there should be a number of switching systems within each EO, MS, or TS. For simplicity, we assume only one switching system within each of them in this paper, and use $L_{i}, M i, T_{i}$ to represent $\mathrm{EO}_{i}, \mathrm{MO}_{i}$, and $\mathrm{TS}_{i}$ respectively.

In view of the demands of the long-distance dialing traffic, in fact, some switching networks connect EOs directly to TSs to reduce the burden on MSs. In our work, the fault isolation in this case will also be considered. Normally, for the regular system checking or failure diagnosis, probe technology is widely used in telecommunication networks where a probe can be made of a phone call with a designated pair of caller and callee. If a phone call is made successfully, we can say that the elements (switches) on the route of that call are healthy (or no problem). Catering for the system functionality of the telecommunication network hierarchy, usually, only EOs can make and receive phone calls in telecommunication networks. So, if a system administrator would like to check if the managed hierarchical telecommunication network functions well, he (or she) has to make numerous phone calls to get a comprehensive check for each of the switches on the network. For example, one simple method for achieving the check is to make each EO probe each of the other EOs; if there are $m$ EOs in the managed telecommunication network, then $m(m-1)$ probes would be needed. For a large network, this $\mathrm{O}\left(\mathrm{m}^{2}\right)$ level of probing would be unacceptable due to its large overhead. Thus, figuring out a way to effectively utilize probes to do the fault isolation more efficiently is significant. Please be aware that, in our work, only EOs

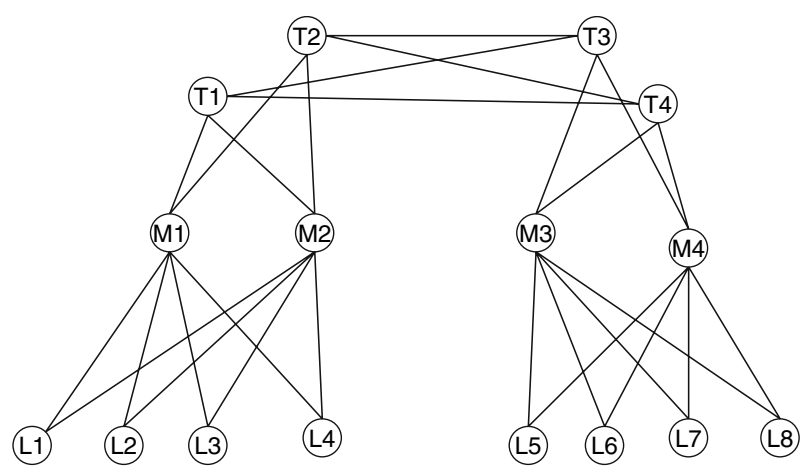

Fig. 1 A conceptual hierarchy for telecommunication switching systems 
can be considered and configured as probe stations which can emit probes (or probe phones).

\subsection{Issues for Active Probing in Telecommunication Networks}

If active probing is used, there would be a number of sets consisting of different combinations of probes which are eligible to do fault isolation for a telecommunication network. During the process of selecting a feasible (or optimal) set of probes, an administrator should take a deep consideration about the following issues for fault isolation in telecommunication networks.

(1) Probe number: fewer number of probes means less effort is needed while doing fault isolation. In addition, smaller number of probes can also imply fewer probe stations should be set up or created, resulting in less effort for probing synchronization among the probe stations. So, finding a probe set with a minimal number of probes is desirable.

(2) Probe synchronization: If there is a probe set which requires multiple probe stations to be deployed in the managed network, there should be an appropriate mechanism to synchronize or coordinate the probing actions among these stations. In reality, for the most part, such synchronization is difficult to achieve due to the heterogeneity of the various vendors' switching systems and because of network propagation delay.

(3) Station functionality: It would be more feasible if an administrator can choose his/her preferable probe stations for fault isolation. This is because not all of the EOs can be configured equally easily as probe stations to emit probes and do some specific management tasks. Plus, fewer probe stations also means less effort on the preparation for the probing. So, it would be more desirable if the administrators can select the EOs, as the probe stations, at their own will in the managed telecommunication network for probing.

(4) Resource requirement: It is always thought that the tasks of network management consume as little system resource as possible. That means not only the numbers of probes and probe stations, but also the total path length of probes should be kept as small as possible. Smaller path length will mean that less trunk bandwidth will be necessary for active probing-based fault diagnosis.

As a result, the goals of our system development for fault isolation in telecommunication networks are (1) to find a minimal (optimal) probe set with acceptable total probe length; (2) probe stations should be as few as possible; (3) the administrators can pick up EOs as probe stations at their will. In the rest of this paper, we will introduce how we utilize call-forwarding feature combined with several well-known active probing mechanisms to facilitate the establishment of an effective and optimal probe set, as required by our goals. 


\section{Active Probing Algorithms for POTS}

In this section, the concepts of active probing are described. Three current widelyused active probing algorithms for fault isolation and the associated matrix-based data structure are also mentioned. On the basis of the concepts of active probing, we apply phone call-forwarding in these algorithms to achieve the goals of our system development for fault isolation in telecommunication networks. Our fault isolation currently focuses on the diagnosis of permanent single-node failure in the managed telecommunication network.

\subsection{Concepts of Active Probing Technology}

Brodie et al. $[10,11]$ use a binary string to represent a probe such that each probe consists of an $n$-bit binary string if there are $n$ nodes in the managed network. The value of the $j$ th bit of a probe would be set to 1 if the $j$ th node is passed through by that probe; otherwise the bit is 0 . The set of these probes can form an $r$-by- $n$ matrix called dependency matrix where $r$ and $n$ are the number of probes and nodes, respectively. For instance, Fig. 2 is the dependency matrix of an example probe set of six probes for Fig. 1. In Fig. 2, probe P17 at the first row with the value of (10000010100110100) indicates that it goes through nodes L1, M1, T1, T3, M4, and L7. Please note that (1) column $\mathrm{Z}$ is used for representing the condition of no fault occurrence; (2) only $\mathrm{EO}_{i}\left(\mathrm{~L}_{i}\right)$ can emit probe phone calls in the telecommunication networks; (3) how the probe set of Fig. 2 comes from will be explained soon in Sect. 3.2.

Given the dependency matrix of a probe set, we can calculate the diagnostic power of that set. Let $\mathrm{S} 0=\left\{n_{1}, n_{2}, \ldots, n_{i}\right\}$ be the initial set of nodes. If $\left\{n_{1}, n_{2}, \ldots\right.$, $\left.n_{i}\right\}$ can be decomposed into $\left\{\left\{n_{1}\right\},\left\{n_{2}\right\}, \ldots,\left\{n_{i}\right\}\right\}$ by using a given set of probes, we can say that the failure of each node can be isolated or identified. For the example in Fig. 2, six probes are used to probe a network of sixteen nodes. The first probe, P17, passes through L1, L7, M1, M4, T1, and T3, inducing the decomposition $\mathrm{S} 1=\{\{\mathrm{L} 1, \mathrm{~L} 7, \mathrm{M} 1, \mathrm{M} 4, \mathrm{~T} 1, \mathrm{~T} 3\},\{\mathrm{L} 2, \mathrm{~L} 3, \mathrm{~L} 4, \mathrm{~L} 5, \mathrm{~L} 6, \mathrm{~L} 8, \mathrm{M} 2, \mathrm{M} 3, \mathrm{~T} 2, \mathrm{~T} 4, \mathrm{Z}\}\} . \mathrm{In}$ this decomposition, failures which occur at two nodes with mutually exclusive failure durations can be distinguished by the probe set if and only if the nodes belong to different partitions in the decomposition. It means that P17 can be utilized

\begin{tabular}{|c|c|c|c|c|c|c|c|c|c|c|c|c|c|c|c|c|}
\hline L1 & L2 & L3 & L4 & L5 & L6 & L7 & L8 & M1 & M2 & M3 & M4 & $\mathrm{T} 1$ & $\mathrm{~T} 2$ & T3 & $\mathrm{T} 4$ & $\mathrm{Z}$ \\
\hline 1 & 0 & 0 & 0 & 0 & 0 & 1 & 0 & 1 & 0 & 0 & 1 & 1 & 0 & 1 & 0 & 0 \\
\hline 0 & 1 & 0 & 0 & 0 & 0 & 0 & 1 & 1 & 0 & 0 & 1 & 1 & 0 & 1 & 0 & 0 \\
\hline 0 & 0 & 1 & 0 & 0 & 1 & 0 & 0 & 0 & 1 & 1 & 0 & 0 & 1 & 1 & 0 & 0 \\
\hline 0 & 0 & 1 & 0 & 0 & 0 & 0 & 1 & 0 & 1 & 0 & 1 & 0 & 1 & 1 & 0 & 0 \\
\hline 0 & 0 & 0 & 1 & 1 & 0 & 0 & 0 & 0 & 1 & 1 & 0 & 1 & 0 & 1 & 0 & 0 \\
\hline 0 & 0 & 0 & 1 & 0 & 0 & 1 & 0 & 0 & 1 & 0 & 1 & 0 & 1 & 0 & 1 & 0 \\
\hline
\end{tabular}

Fig. 2 The dependency matrix 
to identify the failure is in $\{\mathrm{L} 1, \mathrm{~L} 7, \mathrm{M} 1, \mathrm{M} 4, \mathrm{~T} 1, \mathrm{~T} 3\}$ or in $\{\mathrm{L} 2, \mathrm{~L} 3, \mathrm{~L} 4, \mathrm{~L} 5, \mathrm{~L} 6, \mathrm{~L} 8$, M2, M3, T2, T4, Z\}, when a single-node failure occurs. If P17 fails, the failure would be in $\{\mathrm{L} 1, \mathrm{~L} 7, \mathrm{M} 1, \mathrm{M} 4, \mathrm{~T} 1, \mathrm{~T} 3\}$; if not, the failure would take place on one element of $\{\mathrm{L} 2, \mathrm{~L} 3, \mathrm{~L} 4, \mathrm{~L} 5, \mathrm{~L} 6, \mathrm{~L} 8, \mathrm{M} 2, \mathrm{M} 3, \mathrm{~T} 2, \mathrm{~T} 4, \mathrm{Z}\}$. Then the second probe P28 can decompose S1 into S2 $=\{\{\mathrm{L} 1, \mathrm{~L} 7\},\{\mathrm{M} 1, \mathrm{M} 4, \mathrm{~T} 1, \mathrm{~T} 3\},\{\mathrm{L} 2, \mathrm{~L} 8\},\{\mathrm{L} 3, \mathrm{~L} 4$, L5, L6, M2, M3, T2, T4, Z \} \} in the same fashion. Next, P36 which passes through L3, L6, M2, M3, T2, and T3 decomposes S2 and yields $\{\{\mathrm{L} 1, \mathrm{~L} 7\},\{\mathrm{M} 1, \mathrm{M} 4, \mathrm{~T} 1\}$, $\{\mathrm{T} 3\},\{\mathrm{L} 2, \mathrm{~L} 8\},\{\mathrm{L} 3, \mathrm{~L} 6, \mathrm{M} 2, \mathrm{M} 3, \mathrm{~T} 2\},\{\mathrm{L} 4, \mathrm{~L} 5, \mathrm{~T} 4, \mathrm{Z}\}\}$. Continuing in this way, the probe set in Fig. 2 has a diagnostic power of $\{\{\mathrm{Z}\},\{\mathrm{T} 4\},\{\mathrm{L} 5\},\{\mathrm{L} 4\},\{\mathrm{L} 6\}$, $\{\mathrm{M} 3\},\{\mathrm{L} 3\},\{\mathrm{T} 2\},\{\mathrm{M} 2\},\{\mathrm{L} 2\},\{\mathrm{L} 8\},\{\mathrm{L} 1\},\{\mathrm{L} 7\},\{\mathrm{M} 1\},\{\mathrm{T} 1\},\{\mathrm{M} 4\},\{\mathrm{T} 3\}\}$ for the network in Fig. 1, meaning it can isolate each node's failure in that network.

Given a probe set with the single-failure diagnostic power, now let us see how we can use the probe set to do fault isolation. For the example of Fig. 2, probes P36 and P54 will not succeed if node M3 fails. In this case, first, we get complementary values of P17, P28, P38, and P74, where these are the probes that are successful. Then we execute the logic AND operation on all of the probes in the matrix, i.e., $\overline{P 17} \cdot \overline{P 28} \cdot P 36 \cdot \overline{P 38} \cdot P 54 \cdot \overline{P 74}$ and find that the resulting value of this operation would be 00000000001000000 (the 11th bit is 1), indicating the failure of M3 (the 11 th node on the matrix table). Considering another case of no failure occurring, logic AND operation of $\overline{P 17} \cdot \overline{P 28} \cdot \overline{P 36} \cdot \overline{P 38} \cdot \overline{P 54} \cdot \overline{P 74}$ would be carried out and the resulting value is 00000000000000001 (indicating $\mathrm{Z}$ fails), where node $\mathrm{Z}$ stands for the occurrence of no faults. With these concepts mentioned above, the following subsection will introduce three well-known algorithms widely used to figure out the optimal or close-to-optimal probe set which consists of the least number of probes and has the single-failure diagnostic power. Please notice that a dependency matrix has single-failure diagnostic power for fault isolation only if each column is unique in the dependency matrix table [10].

\subsection{Active Probing Approaches for Fault Isolation}

Brodie et al. proposed three different algorithms for finding a probe set for fault isolation: Exhaustive search is an optimal algorithm which finds the minimal probe set, while subtractive search and additive search are heuristic algorithms which have polynomial complexity but may not always find the minimal probe set. In our work, these three algorithms are considered for adaptation to the fault isolation of telecommunication networks. Before doing that, let us first check into the approaches used by these three algorithms.

The minimal probe set can of course be found by exhaustive search. Basically, there would be $\lceil\log (n)+1\rceil$ probes at least to isolate the single-node failure for a network of $n$ nodes. Thus, only probe subsets of size between $\lceil\log (n)+1\rceil$ and $n$ are needed to be considered. This algorithm initially checks each of the probe subsets of size $\lceil\log (n)+1\rceil$ to see if any one of them has the same diagnostic power as the original set. If none can be found, the process further checks each of the probe subsets of size $\lceil\log (n)+2\rceil$. The process proceeds continuously in this manner until either a subset is found to have the equivalent diagnostic power or none can be 
found after checking the last one of the probe subsets of size $n$. This algorithm is proved to have a computational complexity of $\mathrm{O}\left(k^{n}\right)$, with the assumption that each node has at least $k$ distinct probes through it and there are $n$ nodes on the probed network [10]. This is clearly prohibitive unless the network is quite small.

Subtractive search starts with the initial set of $r$ probes, considers each probe in turn, and discards it if it is not needed; i.e., if the diagnostic power remains the same even if it is dropped from the probe set. This algorithm terminates in a subset with the same diagnostic power as the original set but which may not necessarily be of minimal size. The running time is linear in the size of the original probe set, because each probe is considered only once; this gives a computational complexity of $\mathrm{O}(r)$.

Another approach is a greedy search algorithm where at each step we add the probe that results in the "most informative" decomposition. For instance, suppose probe set P1 induces the decomposition $\mathrm{S} 1=\left\{\left\{n_{1}, n_{2}\right\},\left\{n_{3}, n_{4}\right\}\right\}$ while probe set P2 induces the decomposition $\mathrm{S} 2=\left\{\left\{n_{1}\right\},\left\{n_{2}, n_{3}, n_{4}\right\}\right\}$. Although $\mathrm{P} 2$ can uniquely diagnose one of the nodes and P1 cannot, it might be possible to add just a single probe to P1 and thereby diagnose all the nodes, whereas at least two additional probes must be added to P2 before all the nodes can be diagnosed. Thus, S1 is a more "informative" decomposition than S2. In name only, additive search performs a reverse process compared with that of the subtractive search. It starts with the initial set of no probes, considers the most "informative" probe estimated by employing a conditional entropy-based calculation $[10,11]$ at each run, and includes it into the probe set. This process terminates in a subset with the same diagnostic power as the original set. According to the experimental results, additive search can achieve the optimal (minimal) probe set in most of the cases. The running time of this algorithm is quadratic in $r$, the size of the original probe set, because at each step the information content of the decomposition induced by the newly added probe must be computed.

\subsection{Active Probing with Call-Forwarding in Telecommunication Networks}

In addition to the issues indicated in Sect. 2.2, before the probing approaches which are proposed by Brodie et al. are applied to fault isolation of telecommunication networks, two more issues should be taken into account:

(1) The minimal (or optimal) probe set obtained by these approaches may not work well on telecommunication networks. For example, suppose there is a minimal probe set with six probes starting from six different probe stations. As mentioned previously, synchronizing these six probe stations to cooperatively probe would be a challenge.

(2) The probe stations needed for the minimal probe set induced by those approaches might not be capable of emitting probes in telecommunication networks, i.e., they might not be EOs. Only EOs can make, receive, and forward the phone calls.

Considering the issues and goals of our system development for fault isolation in telecommunication networks, the call-forwarding [12] feature available in these 
networks is adopted to facilitate our active probing. Call-forwarding is a gospel to the mobile workers who need to travel and work everywhere. People can use it to transfer a phone call from one place to another which can reach them; of course, this functionality can lengthen the active phone probes. This implies that a smaller probe set with the same diagnostic power and the designation of specific probe stations can be achieved via the call-forwarding functionality. If so, the impact of synchronization problems can accordingly be lessened.

To do so, first, we view the forwarded phone call probes as general probes which are not forwarded. The example telecommunication network in Fig. 1 will serve to illustrate this concept. Since only EOs are permitted to probe and forward phone calls, for the network in Fig. 1, there would be totally 56 un-forwarded probes (i.e., $m(m-1)$, where $m$ is the number of probe stations) where each EO emits probes to the other seven EOs and self-probing for the probe stations themselves are not counted. If one-call-forwarding is allowed, i.e., phone calls (including probes) can be forwarded at most once, $56+56 * 7$ probes $(56 * 7$ probes are forwarded once; e.g., L1 $\rightarrow \mathrm{M} 1 \rightarrow \mathrm{L} 2 \rightarrow \mathrm{M} 2 \rightarrow \mathrm{L} 3$ in which node L2 is configured call-forwarding to join the probes $\mathrm{L} 1 \rightarrow \mathrm{M} 1 \rightarrow \mathrm{L} 2$ and $\mathrm{L} 2 \rightarrow \mathrm{M} 2 \rightarrow \mathrm{L} 3$ is one of them) can be used for fault isolation. Accordingly, it can be seen that $r=m \sum_{k=1}^{f}(m-1)^{k}$ probes can be produced where $f$ is the maximal phone-forwarding number allowed in the managed telecommunication network. Please notice that the setup of this maximal phoneforwarding number is essential for general telecommunication networks due to some security reasons, such as preventing the occurrence of phone-forwarding loops and fraud dialing [13].

Next, we use the dependency matrix composed of these $r$ probes to figure the optimal set with the three algorithms described in Sect. 3.2 to isolate the single-node failure. However, it is not efficient to input this dependency matrix of size $r$-by$(n+1)$ directly to the algorithms, especially when the network becomes large and complex (ex., in Fig. 1, $r=313712$ and $n=17$ ). Before inputting the dependency matrix into the algorithms, some redundancy should be removed beforehand. For example, $\mathrm{L} 1 \rightarrow \mathrm{M} 1 \rightarrow \mathrm{L} 2 \rightarrow \mathrm{M} 2 \rightarrow \mathrm{L} 3$ and $\mathrm{L} 1 \rightarrow \mathrm{M} 1 \rightarrow \mathrm{L} 2 \rightarrow \mathrm{M} 2 \rightarrow \mathrm{L} 3 \rightarrow \mathrm{M} 1 \rightarrow \mathrm{L} 2$ can result in the same binary strings in the dependency matrix for fault isolation of single-node failure, so that $\mathrm{L} 1 \rightarrow \mathrm{M} 1 \rightarrow \mathrm{L} 2 \rightarrow \mathrm{M} 2 \rightarrow \mathrm{L} 3 \rightarrow \mathrm{M} 1 \rightarrow \mathrm{L} 2$ would be removed from the original probe set if the resulted probe set with a less total probe length is expected. Our approach is outlined as below:

1. Given the network topology and phone routing table, the original probe set of size $r=m \sum_{k=1}^{f}(m-1)^{k}$ can be obtained, where $f$ is the max call-forwarding number for a phone call and $m$ is the number of probe stations.

2. Check the original probe set to remove the redundancy of probes which have the same probe binary string. The removal of redundant probes can be based on different requirements or criteria, such as probe length or probe station.

3. Use the trimmed probe set of step 2 to form the dependency matrix required and render the matrix to the three algorithms mentioned in Sect. 3.2 to check their performance for single-node failure isolation in telecommunication networks. 


\section{Experimental Results and Analyses}

In this section, to check the fault isolation performance of exhaustive search, subtractive search, and additive search along with the call-forwarding, experiments are conducted in our simulation networking environment. From several different perspectives, we analyze their corresponding fault isolation performance based on the results of experiments and propose our suggestions.

\subsection{Experimental Networking Environment}

To check the fault isolation performance of these algorithms with the ability of callforwarding, the diagrams for telecommunication networks of both Taichung City (Fig. 3(a)) and Taichung County (Fig. 3(b)) in Taiwan are provided. The corresponding phone call-routing of these two networks are also associated and depicted in the diagrams as (1) a single-arrowed line represents that only one-way traffic can travel between the two nodes which are connected by the arrowed line (2) whereas a double-arrowed dotted line represents two-way traffic is allowed.

As shown in Fig. 3, (a) presents an ordinary hierarchical telecommunication network while Fig. 3(b) represents a mesh-like topology. With the consideration of reducing the burden of MSs $\left(M_{i}\right)$, in Fig. 3(b), the EOs $\left(L_{i}\right)$ deliver the long-distance calls which they get directly to TSs $\left(T_{i}\right)$. No doubt the configuration in Fig. 3(b) can carry out a certain level of fault tolerance; for example, the long-distance phone calls can still work even if $M_{i}$ 's are out of order whereas they cannot work at all for Fig. 3(a) for the same case. More than one hundred and twenty experiments with the three algorithms mentioned in Sect. 3.2 and the two example telecommunication network topologies were performed from March 14, 2006 to July 1, 2006 in our simulation networking environment [14], to verify their fault isolation performance along with call-forwarding. In each experiment, one of the three algorithms was used with the input of the trimmed call forwarding-embedded dependency matrix derived from one of the two network topologies. The same experiment was repeated twenty times to get the average value of measurements. The next subsection will discuss the experiment results from several different perspectives. By the way, in our work, the maximum call-forwarding number allowed in our experiments is bounded at five to conform to the current system configuration in reality, but we vary it from 0 to 5 to observe its impact on the probe set size. A value of 0 corresponds to no call-forwarding.

\subsection{Performance Analyses and Discussions}

Figure 4(a, b) show the size of the minimal probe set on average induced by the three algorithms with different maximal call-forwarding numbers for the networks in Fig. 3(a, b), where (1) the horizontal axis consists of different maximal callforwarding numbers, (2) the vertical axis represents the average size of the resulting minimal probe set, and (3) the probe sets induced by these algorithms are all capable 


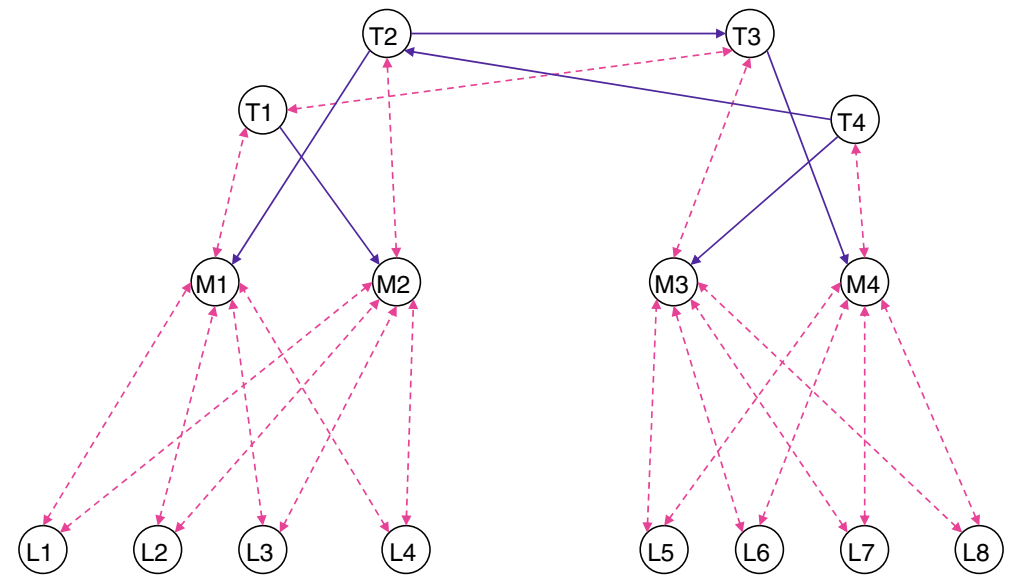

(a)

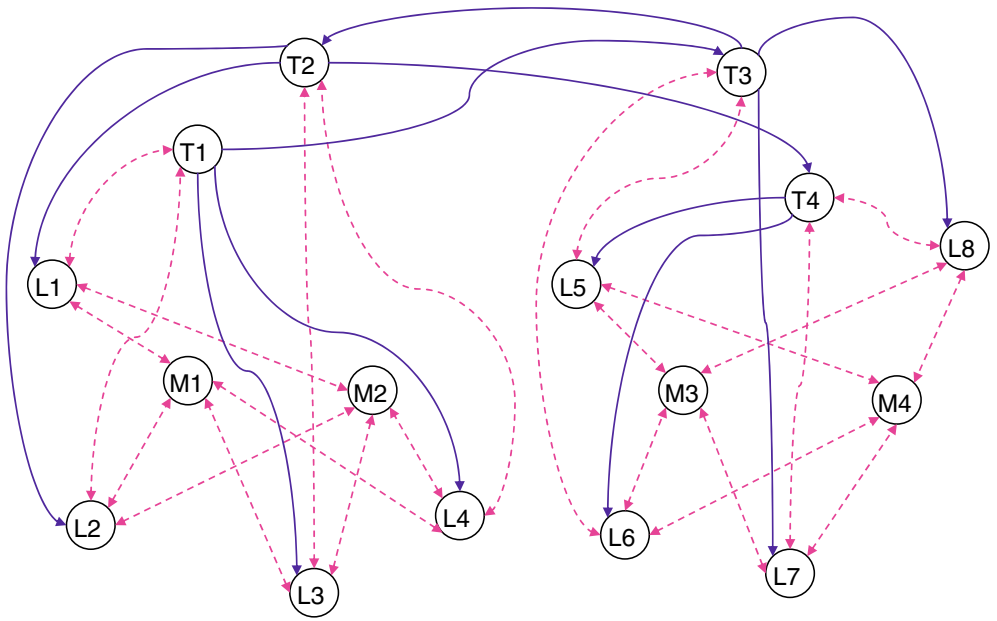

(b)

Fig. 3 Diagrams for telecommunication networks of (a) Taichung City and (b) Taichung County

of isolating single-node failure. The corresponding size of the trimmed original probe set as algorithm input for different maximal call-forwarding numbers is listed on Table 1. As shown in Fig. 4, for exhaustive search and additive search, the size of the minimal probe set can be reduced with the support of call-forwarding. But, it would have no change when the maximal call-forwarding number is larger or equal to two for all three algorithms. Moreover, the subtractive search results in nearly twice the size of the probe sets which are induced by the other two algorithms and keeps it all the same no matter how large the call-forwarding number is. As a result, additive search can be found to have almost the same isolation ability as exhaustive search has (probe set of size $\lceil\log (n)+1\rceil$ ), but only needs polynomial execution time for that. 


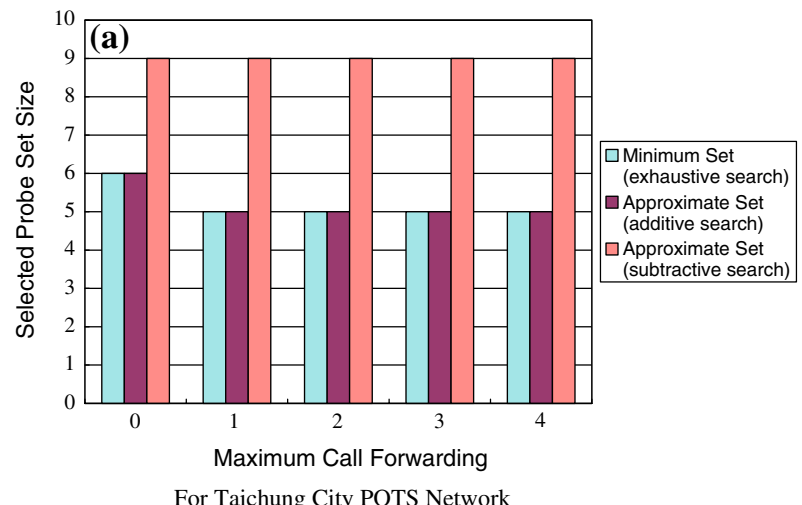

For Taichung City POTS Network

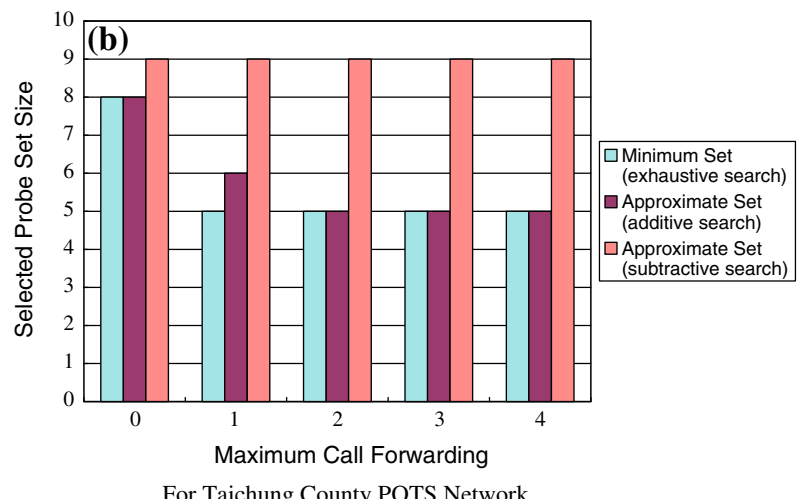

Fig. 4 Isolation performance with call-forwarding

Table 1 Trimmed probe set size versus maximum call forwarding number

\begin{tabular}{llllrrr}
\hline Maximum call forwarding number & 0 & 1 & 2 & 3 & 4 \\
\hline The trimmed original probe set & Taichung City NW & 48 & 268 & 772 & 1388 & 1791 \\
& Taichung County NW & 48 & 304 & 1197 & 3182 & 5433 \\
\hline
\end{tabular}

Now, for eliminating the synchronization problems among probe stations, let us check the possibility of only one EO employed as the probe station in the managed telecommunication network. To do so, at the step 2 of our approach, we will add the following additional heuristics

1. Keep the probes starting from the designated probe station and remove the probes which have the same binary probe code as the ones selected.

2. If two probes start from the same designated probe station and have the same probe code, keep the shorter one to minimize the overall cost (or length) of the resulting probe set. 
Figure $5(\mathrm{a}, \mathrm{b})$ show the performance in which $\mathrm{L} 1$ is employed as the only one probe station for Fig. 3(a, b), and Table 2 lists the corresponding size of the trimmed probe set as algorithm input for different maximal call-forwarding numbers. No results are shown in this figure for maximum call-forwarding value of 0 , because it is impossible to achieve complete coverage for fault isolation with only one probe station when no call-forwarding is used. To further verify this, we performed 60 more experiments with one single probe station on different EO at each trail [15] and found the same results as shown in Fig. 5. Please notice that additive search still performs quite well in this case.

Figure 6 shows the average execution time of these three algorithms with various maximum call-forwarding numbers for the two different topologies used throughout this paper. Again, in Fig. 6, additive search shows good performance on system execution time even though there is some difference between additive search and subtractive search; yet, unquestionably, both of them perform far better than exhaustive search does in this respect. One more thing is worth being noticed: it is still possible that the only one probe station (at one EO) is employed and this EO fails. In such a case, it would cause the entire functionality failure of fault isolation. To avoid this, some important criteria while selecting an EO as the only probe station should be considered and used, e.g. EO's processing power, storage capacity, resistance to failures, accessibility by network administrators, bandwidth capabilities, and administratively designated properties.

\subsection{Further Analyses and Suggestions}

As for the total length of the probe sets induced by the three algorithms, Fig. 7(a, b) show the corresponding experimental results of Fig. 4(a, b). In Fig. 7, we find additive search has at most an increment of around $28 \%$ at the total length of probe sets as the maximum call-forwarding number increases whereas exhaustive search has a total length increment of more than $100 \%$. Meanwhile, both additive search and subtractive search perform well in the aspect of probe sets' total length, but the former needs a much smaller size of probe sets. The fact holds true even in the case of only one probe station [15]. It means that the total probe set length caused by the two algorithms are about the same where additive search needs a smaller probe set than subtractive search does; i.e., additive search needs less management resources than subtractive search does for fault isolation.

Table 2 Trimmed probe set size versus maximum call forwarding number for single probe station

\begin{tabular}{lllllll}
\hline Maximum call forwarding number & & 0 & 1 & 2 & 3 & 4 \\
\hline The trimmed original probe set & Taichung City NW & 7 & 42 & 166 & 360 & 522 \\
& Taichung County NW & 7 & 42 & 172 & 375 & 528 \\
\hline
\end{tabular}



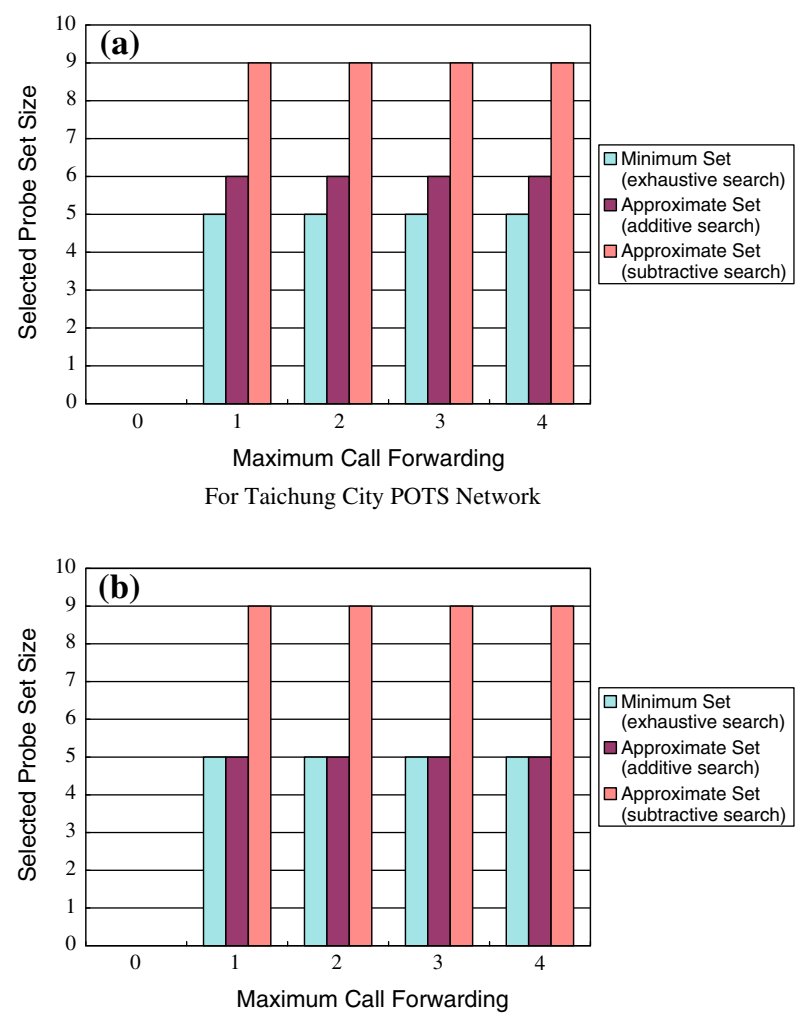

For Taichung County POTS Network

Fig. 5 Isolation performance with call-forwarding and single probe station

According to the experimental results and analyses depicted above, it can be seen that call-forwarding can certainly facilitate fault isolation in telecommunication networks as the issues indicated in this paper are addressed:

(1) Given call-forwarding, the size of the minimal probe set deduced by the Brodie's approaches can generally be reduced. It implies that, with the introduction of call-forwarding, less effort would be needed on the configuration of probe stations and the probing synchronization among them.

(2) By utilizing call-forwarding, it becomes possible to do fault isolation simply with one single probe station, no matter what active probing approach/ algorithm is used. Less probe stations can mean lower possibility of synchronization problems among probe stations and less cost for setting them. On the other hand, without call-forwarding, it can not happen.

In addition, among the algorithms proposed by Brodie et al., we compare them on the basis of the presented performance in this section for reference: 


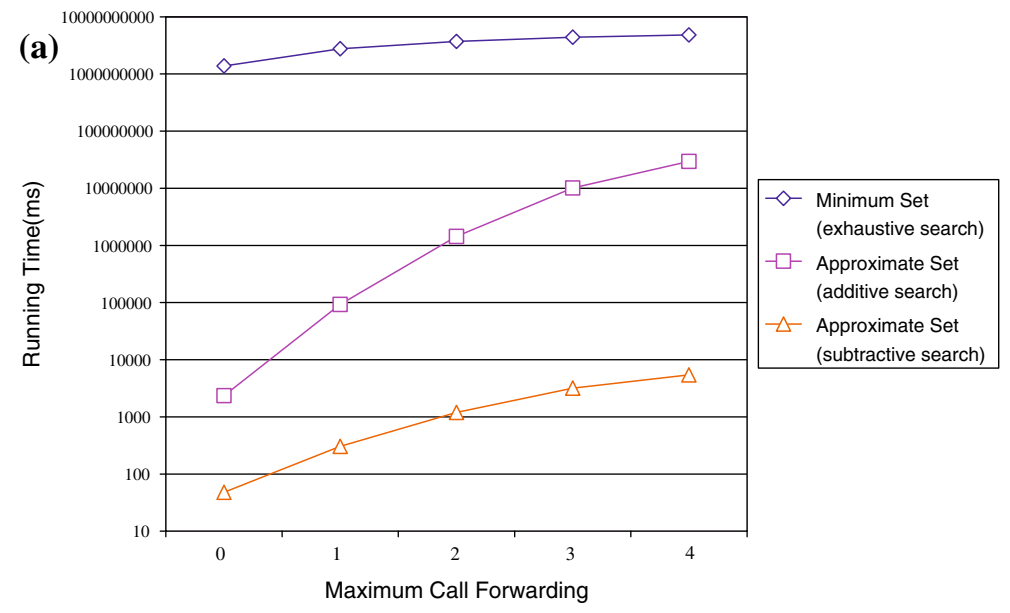

For Taichung City POTS Network

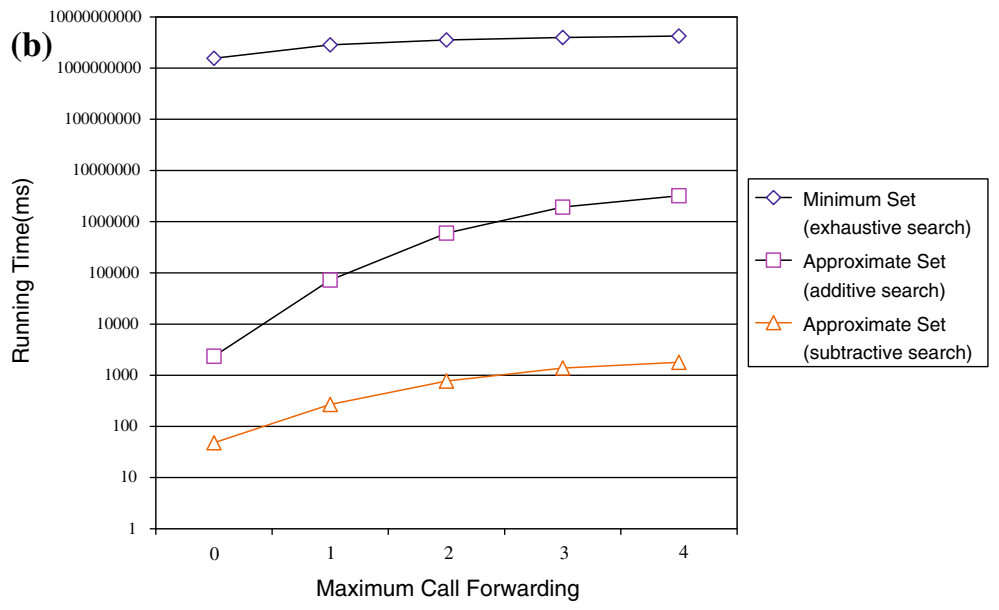

For Taichung County POTS Network

Fig. 6 Algorithm execution time with call-forwarding

(1) Additive search with phone call-forwarding is verified to be most competent to do the fault isolation job in telecommunication networks due to its most efficient and stable performance on this.

(2) Subtractive search with phone call-forwarding can fit the case in which execution time is strictly restricted and no guarantee for the minimal probe set is provided.

(3) Exhaustive search can just serve as an optimalization reference due to its lengthy and unacceptable execution time, even if it can certainly obtain the optimal probe set as expected all the time. 


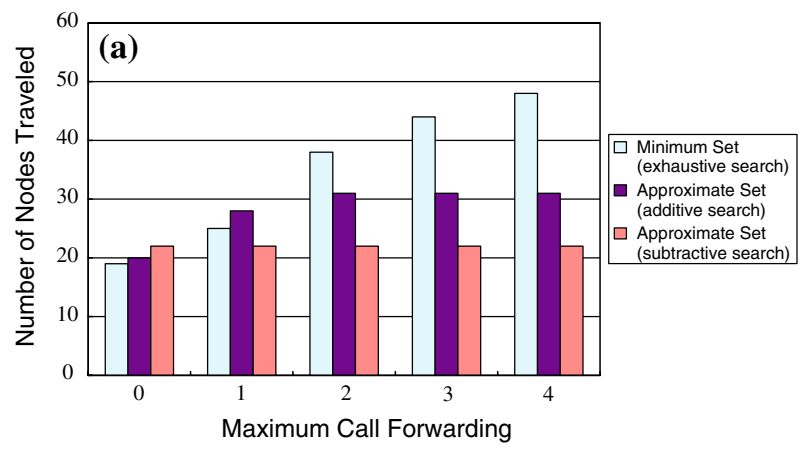

For Taichung City POTS Network

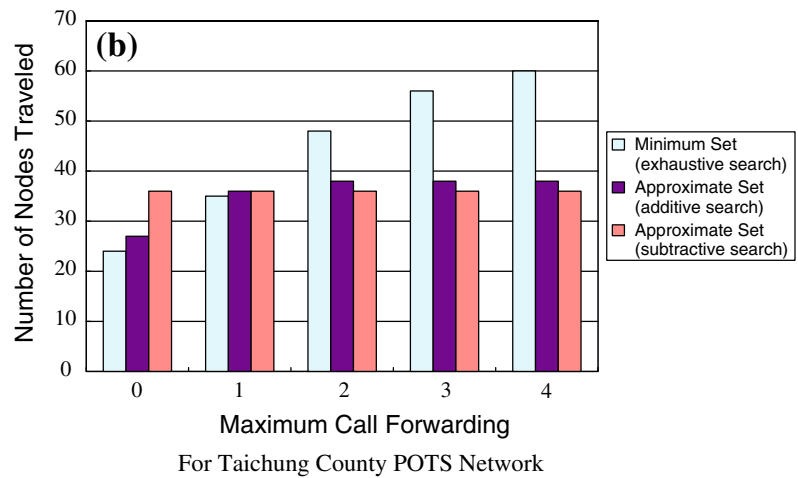

Fig. 7 Probe Set total length with call-forwarding

As a closing of this section, we provide three more suggestions worth being highly noticed while implementing active probing with call forwarding in telecommunication networks:

(1) For the active probing, no matter what kind of networks is or no matter if call forwarding is employed, it can be used for fault isolation (or fault localization) only, not for fault identification. That is, it can not help the users identify what the root fault is and how it occurs.

(2) Given active probing (no matter if call forwarding is employed), it is important for the network managers to well define the probing schedule (or probing frequency). For real-time fault isolation, the frequency of active probing would be higher, but it would result in some adverse effects for the managed network, e.g., excessive network traffic for management purpose.

(3) As for the call forwarding itself, for some specific security purpose, such as avoiding fraud dialing or call looping, the maximum number of call forwarding for a phone call in a managed telecommunication network is usually restricted [15]. This is why the maximum call forwarding number in our fault isolation is limited within four times. 


\section{Conclusion and Future Works}

We have investigated, examined, and tried to adapt the current active probing technologies to fault isolation of telecommunication networks. The unfitness, issues, and difficulties while adopting these IP-networking probing methods into fault isolation of telecommunication networks are enumerated. For achieving our goal, call-forwarding which is a dedicated function in telecommunication networks and has not been considered as part of the POTS fault isolation until now is utilized to overcome the unfitness and difficulties while applying probing technologies to fault isolation of telecommunication networks. Numerous experiments with different probe sets, probe stations, total probe lengths, network topologies, probing algorithms, and so forth are conducted and analyzed to validate our strategy. From the results of our experiments, we can find that phone call-forwarding can not only facilitate the fault isolation with the existing active probing techniques, but also increase the system flexibility on the installation of probe stations. Meanwhile, on the basis of our experimental results, several practical suggestions are also provided for the system implementation to reduce the negative aspects of those issues mentioned throughout this paper. Currently, in addition to applying the research results onto the system development for fault isolation of Taichung County's POTS, we have been trying to use a much larger telecommunication network, e.g., Taipei City's POTS, as our next example to verify our strategies.

In the upcoming future, we will examine the considerably more complex dependency matrix problem for the current active probing techniques, which is caused by fault isolation of multiple simultaneous faulty nodes (and links) in POTS. In addition, fault isolation in the case of networks equipped with load distribution, load balance, and fault tolerance mechanisms will be taken into account [16]. Lastly, a novel algorithm for the minimal probe set for fault isolation would be desired to deal with these upcoming issues.

Acknowledgement The views and conclusions contained in this document are those of the authors and should not be interpreted as representing the official policies, either expressed or implied of the Army Research Laboratory or the U.S. Government.

\section{References}

1. Steinder, M., Sethi, A.S.: A survey of fault localization techniques in computer networks. Sci. Comput. Program. 53(2), 165-194 (2004)

2. Yemini, S.A., Kliger, S., Mozes, E., Yemini, Y., Ohsie, D.: High speed and robust event correlation. IEEE Commun. 34(5), 82-90 (1996)

3. Katzela, I., Schwartz, M.: Schemes for fault identification in communication networks. IEEE/ACM T. Network. 3(6), 753-764 (1995)

4. Rouvellou, I., Hart, G.W.: Automatic alarm correlation for fault identification. In; Proceedings of IEEE INFOCOM'95, pp. 553-561, Boston, MA (1995)

5. Chao, C.S., Liu, A.C.: An alarm management framework for automated network fault identification. Comp. Commun. 27(13), 1341-1353 (2004)

6. Hood, C.S., Ji, C.: Proactive network management. In: Proceedings of IEEE INFOCOM'97, pp. 1147-1155, Kobe, Japan (1997) 
7. Kirmani, E., Hood, C.S.: Diagnosing network states through intelligent probing. In: Proceedings of IEEE/IFIP Network Operations and Management Symposium (NOMS), vol. 1, pp. 147-160 (2004)

8. Frenkiel, A., Lee H.: EPP: A framework for measuring the end-to-end performance of distributed applications. In: Proceedings of Performance Engineering 'Best Practice' Conference, IBM Academy of Technology (1999)

9. Using Keynote Measurements to Evaluate Content Delivery Networks, available at http://www.avoka.com/resources/keynote/wp_cdn.pdf

10. Brodie, M., Rish, I., Mam, S.: Optimizing probe selection for fault localization. In: Proceedings of the 12th IEEE International Workshop on Distributed System: Operations and Management (DSOM), Nancy, France, May (2001)

11. Brodie, M., Rish, I., Ma, S.: Intelligent probing: a cost-effective approach to fault diagnosis in computer networks. IBM Syst. J. 41(3), 372-385 (2002)

12. ITU-T Recommendation M.3400 (2000), TMN management functions

13. Chang, G.S., Cheng, G.T., Xie, L.T., Huang, Y.X., Su, B.D., Chang, Z.Y.: PSTN network management and control system in Taiwan. Telecommun. J. 34(5), 537-552 (2004)

14. Xiao, R.Z.: A forwarding-based probing technology for POTS fault isolation, Master Thesis, Information Engineering Dept., Feng Chia University (2007)

15. Chao, C.S., Xiao, R.Z., Liu, A.C.: A forwarding-based probing technology for pots fault isolation. In: Proceedings of 2006 Electronic and Communication Applications, vol. 1, pp. 36-44, Taiwan, July 2006

16. Pencole, Y., Cordier, M.O.: A formal framework for the decentralized diagnosis of large scale discrete event systems and its application to telecommunication networks. Artif. Int. 164(1-2), 121170 (2005)

\section{Author Biographies}

Chi-Shih Chao received his MS and Ph.D. degrees in Information Engineering from Feng Chia University, Taiwan, R.O.C. in 1996 and 2001, respectively. From 2002 to 2004, he was the director of Computer Resource Center, OIT, Feng Chia University, and currently he is an assistant professor at the Communications Eng. Dept. of Feng Chia University. He received the Annual Excellent Paper Award from the Computer Society of the Republic of China in 2003, and he is a member of IEEE, ACM, and Phi-Tau-Phi. His research interests include network fault management, telecommunication/computer networks, and network security.

Maitreya Natu received his M.S. degree from the University of Delaware in 2005. He is currently working towards the Ph.D. degree in Computer and Information Sciences at the University of Delaware. His research interests include fault localization for wired and wireless networks, probing techniques for network monitoring and management, and network security problems like intrusions and denial of service attacks.

Adarshpal S. Sethi is a Professor in the Department of Computer and Information Sciences at the University of Delaware, Newark, Delaware, USA. He has an M.Tech. degree in Electrical Engineering and a PhD in Computer Science, both from the Indian Institute of Technology, Kanpur, India. He has served on the faculty at IIT Kanpur, was a visiting faculty at Washington State University, Pullman, WA, and Visiting Scientist at IBM Research Laboratories, Zurich, Switzerland, and at the US Army Research Laboratory, Aberdeen, MD. He is active on the program committees of numerous conferences and on the editorial boards of many journals. His research interests include architectures and protocols for network management, fault management, quality-of-service and resource management, and management of wireless networks. 